\title{
Interview
}

Simon Peng-Keller*, Susanna Meyer Kunz

\section{Seelsorge auf der Covid-19-Intensivstation des Universitätsspitals Zürich}

https://doi.org/10.1515/spircare-2020-0084

Vorab online veröffentlicht 25. Juni 2020

Susanna Meyer Kunz (SMK) ist evangelisch-reformierte Pfarrerin, leitende Spitalseelsorgerin am Universitätsspital Zürich, im ersten Beruf Pflegefachfrau HF, CAS Palliative Care und Notfallpsychologin NNPN, verheiratet und Mutter zweier Töchter. Aktuell ist sie auch verantwortlich für die Seelsorge auf der Covid-19-Intensivstation des Universitätsspitals Zürich. Das Interview führte Simon Peng-Keller (SPK).

SPK: Könntest $\mathrm{Du}$ beschreiben, wie sich die Seelsorge und das Universitätsspital Zürich auf die erste Pandemie-Welle vorbereitet hat?

SMK: Die Handreichung, die Ihr verfasst habt, und andere Leitlinien kamen schnell $\mathrm{zu}$ uns. Ich habe mir dann weitere Gedanken gemacht und den für die betreffende Intensivstation verantwortlichen Arzt darauf angesprochen. Wie ist das eigentlich, wenn jetzt Menschen auf dieser Station an Covid-19 sterben? Wie ist der Abschied vorgesehen? Er sagte mir: „Eigentlich ist da kein Abschied vorgesehen. Die Verstorbenen kommen in diese Body-Bags. Und dann gibt es einen Abschied am geschlossenen Sarg.“ Weil ich weiter Fragen stellte, bin ich schließlich für die interprofessionelle Task-Force angefragt worden, die diese organisatorischen Fragen zu klären hatte. Wie werden die Angehörigen informiert? Wie ist das mit dem Abschied, wenn jemand stirbt? Wie wird Seelsorge einbezogen? Schließlich haben wir eine 18-seitige Richtlinie und ein Merkblatt für den Todesfall erarbeitet. Zumindest in einem engen Zeitfenster ist nun der Abschied am Sterbebett möglich. Die Angehörigen dürfen zu zweit auf die Abteilung kommen und da sein. Von den Pflegenden kam die Idee, ob wir kleine Abschiedsfeiern gestalten würden in der Kirche mit maximal fünf Leuten. Das wurde bislang einmal in Anspruch genommen. Die Angehörigen haben

*Korrespondenzautor: Simon Peng-Keller, Professor für Spiritual Care, Universität Zürich, E-Mail: simon.peng-keller@theol.uzh.ch auch die Möglichkeit, uns telefonisch für Entlastungsgespräche zu kontaktieren.

SPK: Was war Dir ein besonderes Anliegen in diesem Vorbereitungsprozess?

SMK: Es war mir ein Anliegen, dazu beizutragen, dass es nicht zu Situationen kam wie in Bergamo oder Spanien. Ich sagte mir: Ich muss mich dafür einsetzen, dass wir diese Abschiede gestalten können. Das ist wichtig für den Trauerprozess. Man weiß ja, dass es die Trauer sehr erschwert, wenn man Verstorbene nicht mehr sehen kann. Es muss möglich sein, dass die Angehörigen Abschied nehmen können. Das war mein Ziel. Und dafür habe ich mich eingesetzt. Dafür mussten wir mit der Pathologie verhandeln, die Vorbehalte gegen eine Aufbahrung hatte. Schließlich haben wir drei Szenarien entwickelt. Wenn bis $\mathrm{zu}$ fünf Menschen pro Tag sterben, ist nun eine Aufbahrung und ein Abschied auf der Abteilung möglich. Wenn mehr sterben, würde es vom Ablauf her schwierig die Qualität einer würdigen Begleitung aufrecht zu erhalten. Da wären wir wahrscheinlich auf weitere fachliche Unterstützung von außen angewiesen.

SPK: Was ist dann geschehen, als die Pandemiewelle uns erreichte?

SMK: Anfang April gab es einige sehr heftige Tage. Da kamen auch die Ärzte und die Pflegenden an ihre Grenzen. Es gab viele Reanimationen, und man merkte plötzlich: jetzt wird's ernst. Bis dahin hatte ich das Gefühl, es trifft wahrscheinlich nur Risikopatienten. Doch kamen plötzlich diese schlimmen Verläufe. Das machte Angst. Man merkte auch, dass man mit dem Beatmen sehr vorsichtig sein musste.

SPK: Kannst Du an einem Beispiel erzählen, wie Deine seelsorgliche Aufgabe auf der Covid-Intensivstation aussieht?

SMK: Mir kommt ein älterer Mann in den Sinn, dessen Zustand zunächst relativ stabil war, sich dann aber sehr schnell verschlechterte. Ich war in regelmäßigem Kontakt 
mit seiner Ehefrau. Ihre größte Sorge war zunächst, dass sie das Handy von ihrem Mann brauchte, um die Rechnungen zu bezahlen. Da konnte ich eine Brückenfunktion übernehmen. Ich habe mit ihr einen Termin vereinbart, um ihr das Handy zu übergeben. Und so haben wir uns das erste Mal getroffen. Sie hat mir beschrieben, was sie durchmacht. Und dann kam eben dieser Tag. Der Zustand ihres Mannes verschlechterte sich dauernd, und wir waren regelmäßig in telefonischem Kontakt. Ich habe auch Sprachnachrichten von ihr und Freunden überbracht, so wie ich es schon regelmäßig gemacht hatte. Einmal wollte sie auch, dass ich den Psalm 23 betete. Sie war reformiert, ihr Mann aus der Kirche ausgetreten und ohne Bekenntnis. Dann kam die Situation, dass man ihr telefonisch mitteilte, dass die Organe versagten und man die Therapie einstellen müsse, dass ihr Mann sterben würde. Es war anspruchsvoll für sie zu entscheiden, ob sie kommen wollte oder nicht, und wir telefonierten mehrfach und ich ermutigte sie zu kommen. Schließlich entschied sie sich dazu, und war dann im Nachhinein sehr froh über diesen Entscheid. Dann gingen wir hinein. Sie war ja noch nie da. Wie bei anderen Angehörigen habe ich ihr geholfen, die Schutzkleidung anzuziehen und bin mit ihr ans Bett ihres Mannes gegangen. Sie konnte ihn dort auch berühren, was ich selbst nie gemacht habe. Mir ist noch in den Ohren, dass sich die Pflege entschuldigte, weil sie keine Kerzen hatten. Am Sterbebett ihres Mannes hat die Frau von ihren gemeinsamen Erfahrungen erzählt. Auch die Söhne, die im Ausland leben, haben wir noch zugeschaltet. Und sie konnten so ihrem Vater noch etwas zum Abschied sagen. Religiöse Rituale wollten sie nicht unbedingt, aber sie wünschten ein Gebet. Weil sie mir zuvor erzählt hatte, dass ihr Mann die Musik von Reinhard Mey liebte, habe ich ihr ganz intuitiv vorgeschlagen, wir könnten doch „Über den Wolken muss die Freiheit wohl grenzenlos sein" singen. Zwei Assistenzärzte haben mitgesungen. Es war eine sehr dichte Atmosphäre. Dann hab ich sie wieder hinaus begleitet und noch einen Moment mit ihr verbracht. Wir sind in die Spitalkapelle gegangen und haben dort eine Kerze angezündet. Wir sind einfach noch einen Moment dort gesessen. Dann habe ich sie wieder hinaus begleitet. Danach haben wir noch einige Male telefoniert. Sie wollte noch etwas für das Team tun und hat einen Kuchen bestellt. Auch die Abteilung hat ihr noch einen Brief geschrieben.

SPK: Konntest Du wahrnehmen, wie die beatmeten Patienten selbst diese Situation erleben?

SMK: Ich erinnere mich an einen Patienten, der einer Freikirche angehörte. Die Angehörigen haben mich gebeten, täglich ein Gebet zu sprechen und auch Audionachrichten geschickt. Einmal hatte jemand aus dem Familienkreis Ge- burtstag. Als ich die Nachricht abspielte, stieg die Pulsfrequenz plötzlich an. Nicht alle Patientinnen und Patienten auf der Intensivstation sind beatmet. Es waren zum Beispiel schwangere Frauen dort. Hier war das Gespräch sehr wichtig, da ja ihre Angehörigen und die anderen kleinen Kinder nicht kommen konnten.

\section{SPK: Wie hast Du das Pflegeteam erlebt?}

SMK: Ich habe immer sehr viel Respekt erlebt. Im Pausenraum habe ich regelmäßig mit den Pflegenden gesprochen. Es ist ja so, dass sie während einer längeren Intensivpflege eine Beziehung zu den Patienten entwickeln und dass sie, wenn jemand gestorben ist, auch traurig sind. Auf der Station selbst bin ich mir oft vorgekommen wie auf dem Mond. Alle stecken in diesen Anzügen. Man erkennt vielleicht die Männer, die etwas größer sind und eine spezielle Haarfarbe haben. Aber sonst sehen da alle gleich aus. Es ist alles gleichförmig und deshalb schwierig, eine andere Atmosphäre herzustellen. Mit der Zeit entstand die Idee, sich wenigstens seinen Namen auf die Schutzkleidung zu schreiben.

SPK: Denkst Du, dass die Pandemie die Arbeit der Spitalseelsorge nachhaltig verändern wird?

SMK: Das ist eine schwierige Frage. Ich weiß es nicht. Wir müssen uns weiterhin sehr aktiv, kreativ und beweglich in das System einbringen. Ich bin überzeugt davon, dass es nicht reicht, wenn wir in der Institution einfach Gäste sind. Es hat sich gezeigt in bestimmten Alters- und Pflegeheimen, in denen Seelsorgende als Gäste betrachtet wurden und nicht mehr eingelassen wurden, zu ungebetene Gäste wurden. Gäste kann man ausladen. Das ist für mich die zentrale Frage, die zu klären ist: Sollen wir zum Behandlungsteam dazugehören? Oder stehen wir außerhalb? Diese Frage muss auch seitens der Kirchen geklärt werden. Es braucht auch eine Verständigung auf Konzepte. Das hat sich in dieser Krisensituation ebenfalls gezeigt. Ich war sehr, sehr froh, dass ich ein Konzept hatte, das ich einbringen konnte, weil auch alle anderen Professionen ihre Konzepte in diese Leitlinie einbrachten. Wir brauchen auch Instrumente wie das Indikationen-Set (Aebi et al. 2019) das sich als sehr hilfreich in dieser Krisensituation erwies.

SPK: Bist Du auf der Covid-Intensivstation Teil des interprofessionellen Teams?

SMK: Ich bin eigentlich im Herzen immer auch noch Pflegefachfrau. Und wenn ich im Spital bin und in einem 
Team arbeite, dann gehöre ich dazu. Aber ich bin eben auch Pfarrerin, Verbi Divini Ministra. Ich denke, wir müssen uns in die Behandlungsteams einbinden lassen. Gleichzeitig finde ich es auch sehr gut, dass ich nicht vom Spital angestellt bin. Das macht mich frei vom ökonomischen Druck. Ich kann dann eine Art Hofnärrinnenfunktion ausüben. Ich glaube, es braucht beides.

\section{SPK: Wie bist Du persönlich mit den Herausforderungen der} letzten Wochen umgegangen?

SMK: Es war mir wichtig, regelmäßig in die Stille zu gehen. Sonst hätte ich das gar nicht geschafft, weil es manchmal schon sehr turbulent und sehr nervenaufreibend und sehr anspruchsvoll ist. Tragend war für mich der Glaube, dass ich in einem großen Ganzen aufgehoben bin, und dass ich in der biblischen Tradition stehe mit ihren
Geschichten und Erfahrungen. Dieses Aufgehobensein hilft mir, meine Aufgabe wahrzunehmen. Zudem war mir bedeutsam, dass ich diese Aufgabe nicht alleine zu bewältigen hatte. Ich bin Teil eines engagierten ökumenischen Teams.

SPK: Ganz herzlichen Dank Susanna, für dieses reiche Gespräch und für Deine Arbeit!

Aebi R, Mösli P, Fankhauser AK, Folini S, Gurtner U, Meier R, Minder H, Schmidt-Aebi M, Wild T, Roser T (2019) Indikationen-Set für Spiritual Care und Seelsorge. Ein Instrument für Gesundheitsfachpersonen zum Beizug der Seelsorge (online). (Zitierdatum 12.05.2020), abrufbar unter http://www.kirchenpalliativebern.c h/fileadmin/user_upload/pdf/Palliative_Care/Indikationen-Se t_Langliste.pdf 